\title{
Topical preparations for pain relief: efficacy and patient adherence
}

\author{
This article was published in the following Dove Press journal: \\ Journal of Pain Research \\ 16 December 2010 \\ Number of times this article has been viewed
}

\author{
Liliana L Jorge ${ }^{1,2}$ \\ Caroline C Feres' \\ Vitor EP Teles ${ }^{3}$ \\ 'Lucy Montoro Institute of \\ Rehabilitation, São Paulo, São Paulo, \\ Brazil; ${ }^{2}$ Albert Einstein Jewish \\ Hospital, São Paulo, São Paulo, \\ Brazil; ${ }^{3}$ Ana Carolina Moura Xavier \\ Rehabilitation Hospital Center, \\ Curitiba, Parana, Brazil
}

\begin{abstract}
There has been an increasing focus on development of new routes of drug administration to provide tailored treatments for patients, without decreasing efficacy of analgesia, in proportion to the progression of the knowledge of pain mechanisms. While acute pain acts as an alarm, chronic pain is a syndrome requiring meticulous selection of analgesic drugs of high bioavailability for long-term use. Such criteria are challenges that topical medications aim to overcome, allowing progressive delivery of active component, maintaining stable plasma levels, with a good safety profile. This review presents recent findings regarding topical formulations of the most widely used drugs for pain treatment, such as nonsteroidal anti-inflammatory agents, anesthetics, and capsaicin, and the role of physical agents as delivery enhancers (phonophoresis and iontophoresis). Although the number of topical agents is limited for use in peripheral conditions, increasing evidence supports the efficacy of these preparations in blocking nociceptive and neuropathic pain. Patient adherence to medical treatment is also a challenge, especially in chronic painful conditions. It is known that reduction of treatment complexity and pill burden are good strategies to increase patient compliance, as discussed here. However, the role of topical presentations, when compared to traditional routes, has not yet been fully explored and thus remains unclear.
\end{abstract}

Keywords: medication adherence, administration, topical, patient compliance, pain, therapeutics

\section{Introduction}

The International Association for the Study of Pain defines pain as a multidimensional entity that involves nociception, afferents to the central nervous system, modulation, affective responses, endogenous analgesia, behavioral adjustments, and changes of social roles. While pain trigger factors are endured, pain degenerates to an independent response, manifesting even when it is possible to eradicate the primary stimulus.

Despite technological advances and thorough established treatments, chronic pain continues to defy health professionals, because it is a poorly controlled condition; ${ }^{1}$ partly because there are three aspects involved in the pathogenesis of pain: nociception (pain sensation and topography), emotional (fear and depression), and behavioral factors (catastrophism, vigilance, and somatic awareness). Pain management faces difficulties that restrict therapeutic success, such as the limited efficiency of analgesics, systemic effects, and cognitive impairment of drugs due to central effects.

As the understanding of pain pathophysiology and treatment increases, new routes of drug delivery are being discovered with the objective of attempting to block pain
Correspondence: Liliana L Jorge Albert Einstein Jewish Hospital, Av. Albert Einstein 665, Block D, 3rd Floor, 05652000 São Paulo, São Paulo, Brazil

Fax +55 I I 30324787

Email ljiliana@ig.com.br 
at peripheral sites, with maximum active drug and minimal systemic effects. Topical preparations are the result of such exploration.

For patients, the rationale is the application of analgesics directly on the site of soreness, even if the sensation is a referred pain from another source. They perceive that oral treatments can potentially lead to more adverse effects as compared to topical treatment. In addition, the intention of applying localized treatment within the lay population may be a means of enhancing the efficacy of the placebo element of topical analgesia. Such mechanisms are of limited value if the area of soreness is large, such as an entire limb in sympathetically maintained pain syndromes, and overall, placebo interventions do not seem to be involved in important clinical effects. Nevertheless, it is well known that placebo interventions may influence reported outcomes, especially pain, nausea, and other subjective complaints that are subject to context. ${ }^{2}$

On the other hand, physicians are aware that chronic pain is a complex syndrome involving peripheral and central factors, justifying the prescription of multimodal therapies and drugs of central action.

Evidence based on empirical practice has suggested that topically applied medications can be almost as effective as those taken orally, with a good safety profile in terms of adverse effects. The ultimate goal that motivates the development of topical preparations is the improvement of patient compliance to medical treatment, by providing efficient pain relief with less central nervous system effects and minimal drug regimen burden. Topical preparations can potentially benefit the pediatric population, whose chronic pain management is just as challenging as in adults.

Topical analgesics or anesthetics are defined as liquids, gels, powders, creams, semisolids, emulsions, patches, foams, or aerosols containing an analgesic or anesthetic agent applied on or around the painful site. Most topical preparations are available as patches, ointments, or creams, and this review will focus on cutaneous applications for adult patients, although some studies in children are also mentioned.

\section{Topical preparations and topical delivery systems}

Among the most widely known drugs with peripheral effects are nonsteroidal anti-inflammatory drugs (NSAIDs), anesthetics, capsaicin, alpha-adrenoreceptor antagonists, and cannabinoids. However, not all medications applied topically on the skin have peripheral action. Topically applied opioids predominantly have a central effect. Therefore, one can didactically distinguish transdermal medication such as buprenorphine and fentanyl and topical drugs acting locally, which have a primary role on the peripheral nervous system and decrease of nociceptive afferents (Figures 1 and 2). ${ }^{3}$

Several adjuvants such as viscosity and permeation enhancers, emollients, and preservatives are added to the active component, with the objective of reducing the drug concentration, increasing the absorption, maintaining the drug at the target site, and warranting less toxicity, less clearance, and greater analgesic effect. ${ }^{4}$ Topical preparations are believed to avoid issues associated with oral or intravenous routes such as gastric disturbances, first-pass hepatic metabolism, and variable serum concentrations. In addition, systemic absorption can be reduced without compromising the desired effect.

However, several items must be considered regarding topical preparations. Marked interindividual variability of skin properties may influence percutaneous absorption and distribution of the drug when applied topically, since the stratum corneum is a barrier that limits penetration of substances. The therapeutic effects also depend on the rate, amount, and depth of penetration into the skin and the potential toxicological hazards of drugs. High doses of sensitizing molecules cannot be administered or diffused into skin, and topical preparations should be water soluble and have an affinity for lipids. Besides, the amount of variability of individual metabolic properties directly influences bioavailability and needed doses or precautions. This is of special interest when a well-established preparation used in an adult population is used for children in an off-label or unlicensed fashion.

Physical agents such as electricity (a system called iontophoresis) and ultrasound (phonophoresis) are progressively used as enhancers to increase permeability and delivery of substances into and through the stratum corneum. Despite their popularity, questions remain regarding definitive treatment.

Iontophoresis is the application of high-voltage pulses to the skin. The method enhances transdermal delivery of specific drugs, migrating charged particles across membranes.

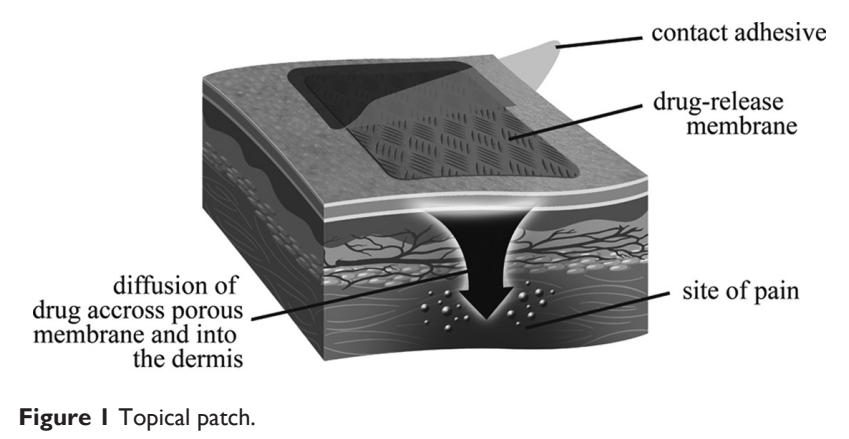




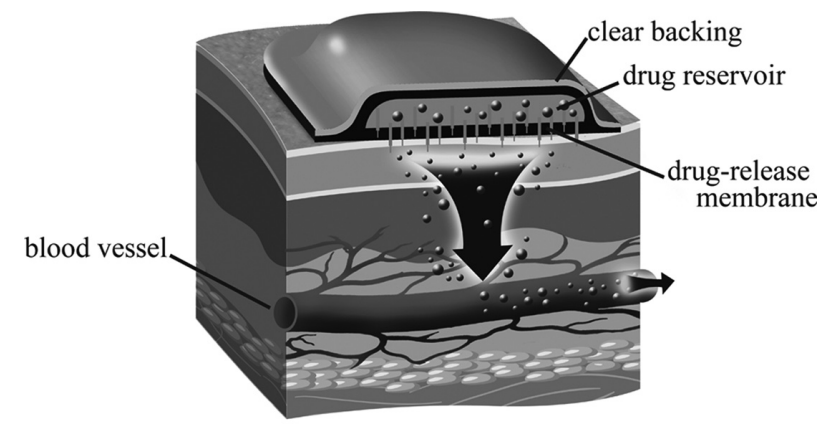

Figure 2 Opioid transdermal patch.

The target can be either local/focal or have systemic effects, and chemical enhancers can be associated. Electrical application is well tolerated in vivo, but can induce muscle contractions. Skin irritation is a common adverse effect.

Phonophoresis is the use of ultrasound waves to enhance drug penetration via heating properties. The effectiveness of ultrasound is reduced when used for muscle spasms or when circulation to the area is disrupted, since its mechanism of action depends on vasodilation and increase of local metabolism. ${ }^{5}$ Phonophoresis at an intensity of $1-3 \mathrm{~W} / \mathrm{cm}^{2}$ and frequency of $1 \mathrm{MHz}$ for $30 \mathrm{~min}$ is able to better enhance ketorolac permeation when compared to $1-2 \mathrm{~W} / \mathrm{cm}^{2}$. Shorter application periods ( $5 \mathrm{~min}$ as described in several protocols) provide nonsignificant effects when compared to ultrasound only. A continuous ultrasound mode is more effective in promoting drug transport than a pulsed mode. However, there are reports of tissue destruction or patient intolerance using 30-min applications with stationary ultrasound. ${ }^{6,7}$

In order to maximize the clinical effectiveness of phonophoresis, skin must be adequately hydrated, preheated, and shaved for ideal ultrasound transmission. Previously applied ultrasound enhances the percutaneous penetration of agents.

A technique associating regional hypothermia and iontophoresis improved bioavailability of sodium diclofenac and prednisolone in synovial fluid. ${ }^{8}$ Other results in animal models suggest the therapeutic antinociceptive effect of diclofenac depends on the current density but not on the application time, and that direct iontophoresis application to the inflamed knee of rats enhanced the therapeutic effect, probably because of direct delivery of the drug. ${ }^{9}$ Both iontophoresis and phonophoresis of naproxen are equally effective electrotherapy methods in the treatment of chronic musculoskeletal conditions, such as lateral epicondylitis. ${ }^{10}$

Other popular devices are biodegradable implantable controlled drug delivery systems, which claim to offer good control of pain, as they continuously apply substances to tissue. Fewer fluctuations of serum levels of drugs are considered to induce less adverse effects with greater patient compliance.

\section{Opioids}

The analgesic effects of opioids are well known, and the two most described are buprenorphine and fentanyl. Despite their transdermal application, the effects are primarily central. Recent research demonstrated the presence of mu and delta opioid receptors at the endings of sensory afferent neurons. Clamping the sciatic nerve in animals caused an inflammatory response, resulting in an accumulation of beta-endorphinergic receptors at both sides of the ligature. In human models, topical morphine promoted relief of mucositis-related pain among patients undergoing head and neck chemotherapy. ${ }^{11}$ However, other studies have failed to demonstrate benefits from the topical use of opioids administered for skin ulcer and dental procedures and painful hypertonic bladder. ${ }^{12}$

\section{Buprenorphine}

Buprenorphine is of special interest, due to its long period of action and antihyperalgesic effects and is free of renal involvement. It is a semisynthetic opioid available in injectable, sublingual, and transdermal presentations (BuTrans). The Buprenorphine patch is used for treatment of moderate to severe cancer pain, but it also seems to induce dosedependent relief for musculoskeletal pain. Demonstration of peripheral opioid receptors in inflamed synovia supports the concept of peripheral opioid analgesia. Indeed, some studies demonstrated the short-term analgesic effect of intraarticular administration of buprenorphine $100 \mu \mathrm{g}$ after knee arthroscopy, whose efficacy was noninferior to bupivacaine $0.25 \%{ }^{13}$ Lower doses of buprenorphine do not produce a similar effect. ${ }^{14}$ Although promising, this area must be elucidated continuously by additional research.

The drug differs from other opioids because of its action as a partial agonist of mu, delta, and kappa receptors and is a complete agonist of opioid receptor-like 1 receptors. ${ }^{15}$ Buprenorphine is antihyperalgesic, partially due to its agonistic effect on kappa receptors, which explains the successful outcome with neuropathic pain and syndromes presenting overt central sensitization. Indeed, there is evidence that neuropathic pain is relatively insensitive to morphine-like mu opioids.

Clinical trials indicate greater pain relief, improvement of sleep quality, and decreased need for rescue therapy, when buprenorphine is used for cancer pain. ${ }^{16}$

Transdermal buprenorphine matrix allows slow release for up to $96 \mathrm{~h}$. Metabolism is independent of the patient's age, and the drug is not immunosuppressive. Buprenorphine does 
not antagonize or cause tolerance when combined with pure microagonists delivered as rescue medication. Its long period of action and relatively safe profile renders buprenorphine as a good option for older patients or those presenting with renal impairment. The analgesic effect is satisfactory for $90 \%$ of patients monitored for 7.5 months. Generally, tramadol, sublingual buprenorphine, or paracetamol combinations are used in clinical practice when additional pain relief is needed. Among these subjects, more than half reported successful analgesia using one patch with one supplementary daily rescue, indicating low occurrence of tolerance.

Initially, buprenorphine use was not widespread, due to safety issues noted in animal studies. Recently, clinical trials demonstrated attractive drug characteristics for its use in chronic pain, such as relative safety with respiratory depression and renal dysfunction, when compared to opioids such as hydromorphone, methadone, morphine, and fentanyl. The most frequently reported side effects (dizziness, nausea, vomiting, constipation, fatigue, and pruritus) are well tolerated with dose adjustment. ${ }^{17}$ No ceiling dose is described, only the limit posed by adverse respiratory depression. There is a lack of antagonism with other pure mu opioids, thus can be combined safely and effectively. The interchange between oral morphine and buprenorphine leads to pain relief in managed doses, but the definitive equipotency ratio has not yet been established. It has been suggested that approximate equianalgesic ratios of transdermal buprenorphine compared to oral morphine and oral oxycodone are around 1:110, and that of transdermal fentanyl to oral morphine are in the range of $1: 75 .^{16}$

Despite the absence of a difference in side effect profiles when comparing transdermal opioids and oral slow-release morphine, the former leads to significantly less constipation rates. $^{18}$

\section{Fentanyl}

Fentanyl is a high-potency synthetic pure agonist opioid, which interacts with mu opioid receptors. The low molecular weight and lipid solubility enable it to be delivered by the transdermal route. Its principal use is for chronic cancer pain, and since the 1990s, it has also been used for some cases of nonmalignant pain. ${ }^{19}$ For functionally impaired patients with nociceptive pain secondary to osteoarthritis who are ineligible to take other drugs, a systematic review suggests opioids are more effective than control interventions. However, side effects can be substantial, considering that the target population is elderly and present with chronic comorbidities. Because risks are higher than benefits, opioids are not recommended for routine treatment of osteoarthrosis, even if pain is severe. ${ }^{20}$

Fentanyl is in the marketplace as a patch (transdermal route, Duragesic ${ }^{\circledR}$; Alza Corporation, Mountain View, CA; Janssen Pharm Inc., Titusville, NJ), nasal spray, inhaler, lollipop, as well as buccal tablet for transmucosal absorption. Transdermal administration provides long-term analgesia, by releasing fentanyl into the bloodstream within 48-72 h, with some variability depending on factors such as skin type, amount of body fat, different manufacturers, and placement of the patch. The patch is available in a variety of sizes and dosages $(12.5,25,50,75$, and $100 \mu \mathrm{g} / \mathrm{h})$. Fentanyl $12.5 \mu \mathrm{g} / \mathrm{h}$ is suitable for moderate pain and the pediatric palliative population.

The nasal route of presentation results in a rapid response, but evidence is scarce and there are concerns related to safety and cost.

The fentanyl membrane patch matrix has a low drug concentration, aiming to increase safety and avoid the fentanyl-containing gel present in traditional fentanyl reservoir patches. This silicon matrix with a controlling membrane contains the drug and provides slow release at a constant rate. Matrix fentanyl was not inferior to gold-standard opioids for pain relief during 30-day follow-up of cancer patients. They reported good tolerance of side effects such as dizziness, somnolence, and constipation. ${ }^{21}$

A patient-controlled fentanyl iontophoretic transdermal system (ITS) (Figure 3) is approved by the US Food and Drug Administration (FDA) for the management of acute postsurgical pain. It provides self pain control to patients without fluctuations in analgesia, in contrast to the traditional fentanyl patch, which releases the drug at fixed rates within $72 \mathrm{~h}$ and was designed for use in chronic pain. The device comprises an electrical field of $170 \mathrm{~mA}$ that actively allows diffusion of ionized fentanyl hydrochloride through intact skin into bloodstream, at a $40-\mu \mathrm{g}$ dose in $10 \mathrm{~min}$. The credit card-sized equipment does not require needles and is preprogrammed, eliminating the risk of bleeding, catheter infiltrations, and management errors. Fentanyl ITS and intravenous fentanyl differ in 1-h plasma concentration, which are 0.1 and $0.7 \mathrm{ng} /$ $\mathrm{mL}$, respectively. ITS absorption is time-dependent within the first $10 \mathrm{~h}$ of dosing. This delivery technology is superior to placebo, and it is equivalent to patient-controlled morphine analgesia (PCA). ${ }^{22,23}$ In pediatrics, one must note that the time to achieve plasma steady state and clearance are longer, and elimination is faster. For children, studies verifying superiority of fentanyl over morphine are rare, and randomized controlled trials in the pediatric population are still lacking. 


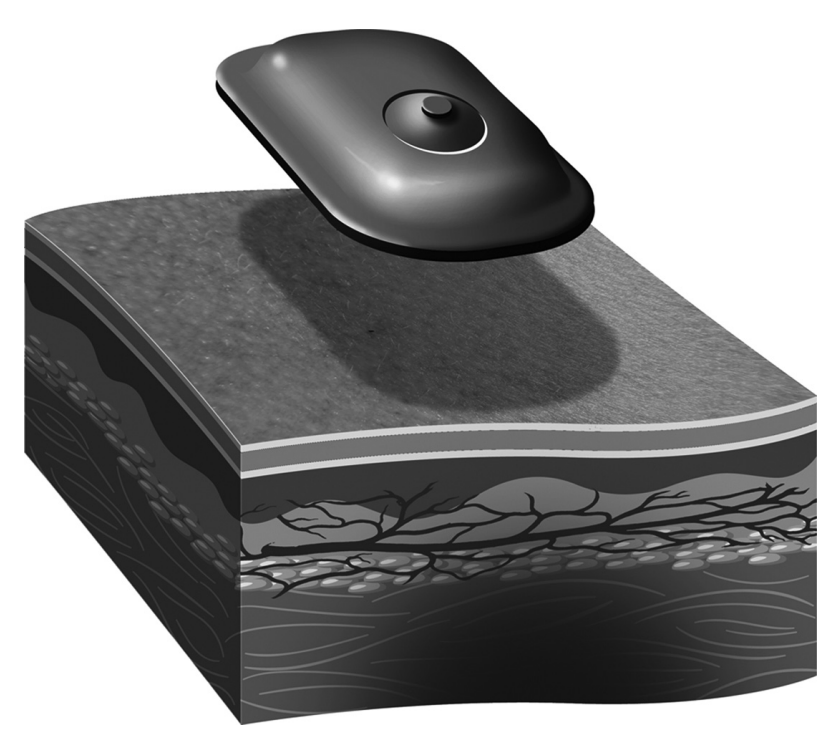

Figure 3 lontophoretical transdermal system (ITS). ITS delivers drug into the bloodstream, and includes: anode hydrogel (ionized drug reservoir), cathode (inert ingredients), battery, and protective plastic liner.

However, the drug is being used off label or unlicensed with increasing frequency as a promising option. ${ }^{24}$ Despite the paucity of clear regulatory guidelines, parents and medical professionals appear to be satisfied with fentanyl ITS.

The transdermal fentanyl system with a drug release rate of $12.5 \mu \mathrm{g} / \mathrm{h}$ matches the lower dosing requirements for pain relief in children. Time to reach steady state serum drug concentrations is longer, clearance is higher, and half-life elimination is shorter in children than in adults. A conversion factor of $45 \mathrm{mg}$ /day oral morphine to $12.5 \mu \mathrm{g} / \mathrm{h}$ transdermal fentanyl is used for initial therapy dose estimation in children receiving long-term morphine therapy. Children may take longer to reach stable fentanyl serum concentrations than adults, and younger children require higher doses than adults. ${ }^{24}$ In an attempt to provide more flexible dosing for pain management in children, some encourage the use of partially occluded fentanyl patches. However, this technique requires further tests in clinical practice. ${ }^{25}$

Adverse effects have been observed with pure opioid agonists. The most common are nausea, vomiting, headache, and erythema, with low rates of respiratory depression. When comparing children and adults, the profile of adverse effects is similar. Increasing doses lead to respiratory impairment, corresponding to plasma concentrations above $1.4-2.5 \mathrm{ng} / \mathrm{mL}$. Excessive use of iontophoretic fentanyl and abuse can lead to death. Fentanyl resulted in less constipation when compared to morphine, both in adults and in children. Strategies to minimize long-term adverse effects of opioids include dose monitoring, opioid rotations, and change of administration routes.

\section{Anesthetics}

Anesthetic creams have been developed to counteract the discomfort related to venipuncture and intravenous catheter insertion in hospitalized children and adults. In fact, blood draws are the most frequently reported painful events.

Despite the clear demand for topical anesthetic drugs in hospital settings and the fact that application of anesthetic creams are painless in comparison to anesthetic infiltrations, the former remains in disuse, partly due to the long onset of action and inconsistent effectiveness reported. For utility in practice, a topical anesthetic must be easy to apply, have few side effects, not require specialized equipment, and have a low cost. Transdermal technologies that promote the flow of several sizes of molecules through the skin barrier via the creation of transient microchannels are able to provide greater anesthesia in $<20$ min. $^{26}$

Many anesthetics are available in gel, cream, or patch. A widely used product is the eutectic mix of lidocaine $2.5 \%$ and prilocaine $2.5 \%\left(\right.$ EMLA $^{\circledR}$ cream-2 ; Astra Zeneca Pharmaceuticals, Wilmington, DE; APP Pharmaceuticals, Schaumburg, IL). It has been employed as a cutaneous anesthetic applied before invasive procedures and empirically for the treatment of postherpetic pain. However, the US Food and Drug Administration has not approved it for treatment of neuropathic pain. No safety studies on long-term use of topical anesthetics were found in the literature. Methemoglobinemia has been associated with prilocaine. There are reports of lidocaine patch and EMLA cream-induced allergic contact dermatitis, suggesting that prilocaine and the vehicles propylene glycol and parabens may act as immunogens.

Ametop $^{\circledR}$ (Smith \& Nephew Healthcare Ltd, London, UK) is a tetracaine base $4 \%$ water-in-water gel that has been used extensively in adults and children for cutaneous pain related to procedures. A meta-analysis of 534 children comparing amethocaine with EMLA that evaluated anesthetic efficacy, ease of needle insertion, and resultant skin changes showed that despite the known efficacy of EMLA as a topical anesthetic for children, amethocaine was superior in reducing pain following needle insertion procedures. ${ }^{27}$ EMLA and Ametop are examples of pediatric evidence being extrapolated for application in adults. However, this translation into adult clinical practice must be done cautiously, because of the known differences of skin thickness, pharmacokinetics, and metabolism between these two populations.

A systematic review verified the efficacy of EMLA plasters for analgesia during debridement or dressings. The mixture provided significant relief, but the effect on tissue healing remains obscure. There is no evidence regarding 
the advantage of the use of EMLA on persistent pain following chronic venous insufficiency. ${ }^{28}$

Recently, a patch containing lidocaine $70 \mathrm{mg}$ and tetracaine $70 \mathrm{mg}$ (Rapydan ${ }^{\circledR}$ in Europe [EUSA Pharma Inc., Oxford, UK] and Synera ${ }^{\circledR}$ in USA [Endo Pharmaceuticals Inc., Ford, PA]) combined with a controlled heat-assisted drug delivery system for fast onset of action has been FDA- and European Medicines Agency-approved for pain relief during needle punctures, cannulations, and other superficial surgical procedures. The drug combination is indicated for surface anesthesia of normal intact skin in children aged above 3 years and in adults. When compared to EMLA in controlled clinical trials, the novel patch provided effective anesthesia with an application time of $10 \mathrm{~min}$. Data suggest that the lidocaine/ tetracaine patch may represent an important alternative to lidocaine/prilocaine cream for topical local anesthesia before vascular access among adults ${ }^{29}$ and children. ${ }^{30}$

Other topical drugs such as a combination of tetracaine, adrenaline, and cocaine (TAC) have been tested for analgesia during procedures in children. However, their use is not supported in the literature because of toxicity and the availability of superior alternative drugs. Other combinations such as lidocaine, epinephrine, and tetracaine (LET), as well as bupivacaine plus norepinephrine, were effective for scalp analgesia during repair of lacerations.

\section{Lidocaine}

Lidocaine blocks voltage-gated sodium channels, and topical administration is believed to hush ectopic discharges of fine afferent fibers. It was recognized that a lidocaine patch acted only on abnormal, intact hyperalgesic afferent receptors. Recently, patches proved to be effective also in skin devoid of nociceptors. ${ }^{31}$ Topical lidocaine slows peripheral nociceptor sensitization and central hyperexcitability. Patients presenting with concurrent allodynia were the most extensively studied in clinical trials, but analgesic benefits were also observed in patients presenting with nonallodynic neuropathic pain.

Lidocaine is available in $5 \%$ patches (Lidoderm ${ }^{\circledR}$; Endo Pharmaceuticals Inc., ) and is approved in the USA for postherpetic neuralgia treatment and other focal neuropathic syndromes (mononeuropathies, intercostal neuralgias, and postamputation pain) in which traditional treatment using tricyclic antidepressants, anticonvulsants, and opioids fail. ${ }^{32-34}$ Nevertheless, topical lidocaine should only be prescribed as a second-line agent for postherpetic neuralgia. ${ }^{35}$

Positive effects on quality of life scales and pain are supported by several clinical trials, where up to three applications were noted in $12 \mathrm{~h}$.
Several pharmacokinetic studies demonstrated that systemic absorption of the lidocaine patch in healthy adults is minimal when up to four units are applied with 12-24-h/day dosing; absorption rate is lower among postherpetic neuralgia patients, who benefit from a pure lidocaine patch or combined with other agents. No drug-drug interaction was observed, and its use does not result in a beta-mediated sensory loss at the application site.

Topical anesthetics (lidocaine 5\% ointment, pramoxine hydrochloride $1 \%$ with hydrocortisone acetate $1 \%$ in water miscible mucoadhesive foam base, alcoholic lignocaine 5\% spray, lignocaine $1 \%-5 \%$ gel, and cinchocaine $2 \%$ ) were studied for the relief of perineal pain after vaginal delivery. However, evidence of effectiveness and long-term effects were inconclusive. ${ }^{36}$

There are reports of increase in skin blood flow and vascular reactivity with the use of EMLA and a warmthproducing lidocaine/tetracaine patch, which could enhance visibility of veins and improve success rate with punctures. Nevertheless, the hyperemic response is transient, with possibly a stronger effect with Rapydan as compared to EMLA. ${ }^{30,37}$

There is anecdotal evidence from an open-label, nonrandomized trial that lidocaine 5\% patches were safe and effective for subacute and chronic low back pain when used daily for 6 weeks. ${ }^{38}$

The side effects were mild and clinically irrelevant, such as skin irritation. ${ }^{39}$ Due to the safety profile, low incidence of systemic effects, and low probability of interactions with other drugs, the lidocaine patch is considered to be a suitable treatment for focal neuropathic pain, with or without allodynia, also for the elderly. ${ }^{40}$

\section{NSAIDs}

NSAIDs are popular drugs and have widespread use for chronic and acute musculoskeletal conditions. They have the advantage of local action without developing central adverse effects and cognitive impairments. Side effects have been well described, although partly neglected.

Within the NSAID class, each drug has a specific tissue distribution and pharmacodynamics. They block the inflammatory cascade and cyclooxygenases (COX) by inhibiting prostaglandin and thromboxane production and lead to reduction in pain, fever, platelet aggregation, and inflammatory response. ${ }^{41}$

The goal of topical NSAIDs is to minimize systemic adverse effects and encourage compliance. Topical NSAIDs are presented as gel, cream, or spray, to penetrate through 
skin, adipose tissue, and muscles, in sufficient dose to induce peripheral effects without plasma concentration.

Comparison and evaluation of topical NSAIDs are difficult due to the diversity of presentations of the marketed drugs and variety of methods used in the trials. Overall, when NSAIDs are applied topically, a high concentration is observed in the dermis and muscles (equivalent to that obtained with oral route), with less gastrointestinal effects. Plasma concentrations are $5 \%-15 \%$ of those achieved by systemic delivery. In joints, evidence is less robust and suggests that topically applied NSAIDs reach the synovia. Either topical penetration or distribution via systemic circulation could be involved; however, the mechanism remains unclear.

A systematic review of topical NSAIDs for acute musculoskeletal conditions (such as strains and overuse-type injuries) studied 3455 subjects and concluded that the preparations can provide good levels of pain relief, without the systemic adverse events associated with oral NSAIDs. Positive outcomes were observed with use of diclofenac, ibuprofen, ketoprofen, and piroxicam. ${ }^{42}$

Substantial data also suggest that topical NSAIDs have pain-relieving properties in chronic rheumatic diseases. Perhaps an important sign of the increasing importance of using topical medication is that the European League against Rheumatism and the International Osteoarthritis Research Society state that topical NSAIDs are preferred over oral NSAIDs for mild-to-moderate pauciarticular hand and knee osteoarthritis, in patients with sensitivity to oral compounds. ${ }^{43}$ In addition, the UK NICE guidelines for knee and hand osteoarthritis recommend use of paracetamol and/or topical NSAIDs over oral NSAIDs, COX2 inhibitors, and opioids. ${ }^{44}$

Many NSAIDS are sold over-the-counter, representing a clear risk for patients. Another major concern regarding topical NSAID use is the risk of overdosing by applying several preparations at the same time.

Ketorolac tromethamine gel solution was tested for phonophoretical transdermal delivery using pulsed ultrasound in an in vitro inflammatory pain model (cellulose and rabbit skin membranes). Antihyperalgesic effects were noted when compared to topical application only. When ibuprofen $5 \%$, piroxicam, and diclofenac were tested via phonophoresis, diclofenac had the smallest flow and permeability coefficient. ${ }^{45}$

\section{Diclofenac}

This drug has antipyretic and anti-inflammatory actions, with greater COX2 inhibition when compared to indomethacin or naproxen. It has been approved for the treatment of rheumatoid arthritis, acute postsurgical pain, and dysmenorrhea, among other conditions. Adverse effects on gastrointestinal and cardiovascular systems are similar to celecoxib. Other effects include allergies, rash, and renal impairment.

It has high absorption, urinary excretion, a half-life of 1-2 $\mathrm{h}$ and can concentrate in synovial fluid after oral administration, which explains long-term effects even when drug concentration in plasma diminishes.

A diclofenac epolamine (DE) 1.3\% hydroxyethylpyrrolidine (Flector ${ }^{\circledR}$; King Pharmaceuticals Inc., Bristol, TN) patch applied locally for 1-2 weeks provides pain relief for musculoskeletal conditions such as ankle sprains, tendonitis, and knee osteoarthritis, in which analgesia occurs $1 \mathrm{~h}$ after the first application and is superior to placebo after $3 \mathrm{~h}$. This means that the patch provided analgesia in a period in which no diclofenac in bloodstream was expected, inferring a local action. Serum concentration of diclofenac is reached 9-12 h after application of the patch, which suggests the presence of reservoir tissue, since the half-life of DE is short. When compared to $75 \mathrm{mg}$ taken orally, bioavailability is $1 \%$, with few side effects. About $2 \%$ of DE is absorbed by the skin and a steady state condition is reached within $5.4 \mathrm{~h}^{46}$

Formulations in diclofenac gel are available for the management of pain and stiffness in osteoarthritis of the knee, such as Voltaren ${ }^{\circledR}$ Gel (diclofenac sodium topical gel 1\%; Smith \& Nephew Healthcare Ltd). Compared to placebo, $1 \%$ diclofenac gel showed improvement in outcomes up to 4 months after treatment. ${ }^{47,48}$ Results were similar with $1.5 \%$ diclofenac gel associated with dimethyl sulfoxide (DMSO), a penetration enhancer (Pennsaid ${ }^{\circledR}$; Mallinckrodt Inc., St. Louis, MO). ${ }^{49}$ In acute musculoskeletal conditions, the DE patch controls pain and inflammatory signs within 3-14 days when compared to placebo. DE is equivalent to diclofenac diethylammonium gel formulation for pain relief. ${ }^{50}$

Topical NSAIDs can be safer than oral consumption, but further studies are needed to compare topical efficacy to other administration and delivery methods, such as iontophoresis or other physical enhancers.

\section{Ketoprofen}

Besides COX inhibition, ketoprofen stabilizes lysosomal membranes and antagonizes bradykinin action. With the exception of the elderly population, the drug has short halflife. Side effects are similar to diclofenac, and ketoprofen is commercially available as a patch or gel.

Patients with rheumatic diseases or trauma who received a ketoprofen patch reported pain relief and functional gain 
superior to placebo. There is good tolerance, and side effects were cutaneous and related to the patch itself, not to the active component. Although plasma levels of ketoprofen are higher when the drug was administered as a 100-mg patch or gel, total bioavailability of the patch was not more than $10 \%$ of the oral dose. Time release of the patch presentation is longer than $24 \mathrm{~h}$, and the drug remains on the subjacent tissue of application throughout this period. Therefore, the high local concentration and low serum level indicated the drug provided local action without systemic side effects. ${ }^{51}$

Gel formulation (Diractin ${ }^{\circledR}$, formerly IDEA-033; Alpharma Inc., Bridgewater, NJ) is recent in the marketplace and is a viscous, aqueous formulation based on ultradeformable, self-regulating carrier. The ketoprofen patch is noninferior to $100 \mathrm{mg}$ oral celecoxib for analgesia of knee osteoarthritis. ${ }^{52}$ Relief lasted for 12 weeks if applied twice daily. ${ }^{53,54}$

\section{Ibuprofen}

Ibuprofen is rapidly absorbed, has renal excretion, and has a short half-life. Its good penetration into synovial fluid provides antirheumatic effects, which may last after the serum concentration decreases. Thanks to greater tolerance and less frequent adverse effects in comparison to aspirin and indomethacin, ibuprofen has been prescribed for those whose gastrointestinal intolerance prevents the use of other NSAIDs. Ibuprofen is recommended, especially for relief of chronic arthritis and pain following physical exercise.

Studies suggest 5\% ibuprofen gel (Nurofen ${ }^{\circledR}$ Gel; Reckitt Benckiser, Slough, UK) has comparable efficacy to $400 \mathrm{mg}$ oral ibuprofen thrice daily for the treatment of acute pain following musculoskeletal injuries. ${ }^{55} \mathrm{~A}$ cream presentation (Dolgit $^{\circledR}$; Merck, Whitehouse Station, NJ) demonstrated improvement of resting pain and pain during gait among patients with knee osteoarthritis and ankle sprains. ${ }^{56}$

\section{Capsaicin}

Capsaicin is derived from hot chilli peppers from the genus Capsicum and has a long history of use in medical practice. It interacts with sensory afferents via vanilloid receptors VR1, which are cation channels from transient receptor potential family. Chronic exposure to capsaicin stimulates and desensitizes these channels. Besides, capsaicin depletes substance $\mathrm{P}$ at nervous afferent endings and transiently decreases the density of nervous fibers on the skin. Capsaicin is available as $0.025 \%$ cream used $3-4$ times/day or $8 \%$ patch for single dose. ${ }^{57}$ Some of the brand names are Zostrix ${ }^{\circledR}$ (Medicis Pharmaceutical Corporation, Scottsdale, AZ), Qutenza ${ }^{\circledR}$
(NeuoguesX, Inc., San Francisco, CA), Capzasin- $\mathrm{P}^{\circledR}$ (Chattem Labs, Chattanooga, TN), and Zostrix-HP ${ }^{\circledR}$.

In a systematic review, the efficacy of capsaicin was tested in 1556 subjects with musculoskeletal or neuropathic pain. Among neuropathic conditions, $57 \%$ of them had significant analgesia using capsaicin $0.075 \%$ in comparison to $42 \%$ of relief with placebo. Among musculoskeletal causes, there was a $38 \%$ improvement with $0.025 \%$ capsaicin patch in comparison to $25 \%$ relief with placebo. The analgesic effect lasted throughout the 4-8 weeks of follow-up. ${ }^{58}$ Such results are similar to the findings of another recent review, where improvement in neuropathic pain occurred within 6-12 weeks with repetitive use of $0.075 \%$ capsaicin cream or a single patch of $8 \%$ capsaicin. ${ }^{59}$

In placebo-controlled studies, topical capsaicin $0.075 \%$ demonstrated significant effect on diabetic neuropathy, postherpetic neuropathy, postsurgical pain, and Guillain-Barré syndrome. ${ }^{60-62}$

The principal side effects were localized and included burning and erythema, which were worse if applied to moist areas. Inhalation of cream may induce respiratory irritation and bouts of sneezing. However, if applied repeatedly on the same area, burning sensation progressively decreased. Local adverse effects were described in one out of three patients, and one out of 10 had to withdraw. ${ }^{58}$ Sensitization is also described with allergy to latex. ${ }^{63}$

Despite the growing use of capsaicin, especially the cream presentation, this trend is limited, because four times a day application and the resulting burning sensation are inconvenient for most of the subjects and thus threaten compliance.

\section{Nitroglycerin}

Traditionally used in coronary artery diseases, nitrate has been demonstrated as potent analgesic and anti-inflammatory agent, due to its actions over several cellular systems and the central nervous system. Endothelium, macrophages, and neutrophils release nitrate in direct proportion to the increase in glutamate.

Nitroglycerin is an organic nitrate that acts via a supply of free radicals and activation of guanylate cyclase, leading to intracellular increase of cyclic GMP and modulation of phosphodiesterases (PDEs 2, 3, 5) and smooth muscle relaxation. Nitrate also activates ATP-sensitive potassium channels and peripheral antinociception.

Symptom relief in angina pectoris patients follows a decrease of cardiac effort and lower consumption of $\mathrm{O}_{2}$ secondary to a decrease in blood pressure. The effect 
on coronary artery dilation seems to be modest and does not justify the symptomatic improvement. Therefore, the nitroglycerin effect in alleviating ischemic pain is explained by independent mechanisms of vasodilation that it induces. ${ }^{57}$

Nitroglycerin is available in the marketplace as Nitro-Dur ${ }^{\mathrm{B}}$ (Key Pharmaceuticals, Kenilworth, NJ), Nitrodisc ${ }^{\circledR}$ (Roberts Pharmaceuticals, GD Searle \& Co., Peapack, NJ), and Transderm-Nitro ${ }^{\circledR}$ (Novartis Pharmaceuticals, East Hanover, NJ). The two forms of topical glyceryl trinitrate available are as follows: 1) Ointment $2 \%$ applied to $2.5-5 \mathrm{~cm}$ of skin each 4-8 $\mathrm{h}$. The effect commences around $30 \mathrm{~min}$ and lasts up to $6 \mathrm{~h}$. 2) Transdermical disc $2.5-15 \mathrm{mg}$ for $12-16 \mathrm{~h} /$ day. The absorption is progressive and plasma levels are constant the entire day, with peak action occurring between 1 and $2 \mathrm{~h}$. To avoid tolerance, suspending therapy for $8 \mathrm{~h}$ a day is recommended. ${ }^{57}$

Some studies verified the efficiency of nitroglycerin in treating rotator cuff lesions. The transdermical disc had a tendency to improve acute pain when compared to placebo, but several biases prevented a more accurate conclusion regarding long-term responses and functional recovery following treatment. ${ }^{64}$ Minor studies evaluated the analgesic benefit of nitroglycerin in conditions such as varicose vein sclerosis or vulvodynia.

Common side effects are headache, flushing, and palpitations. Serious adverse effects such as hypotension, syncope, and transitory ischemic attack are rare. Allergic and/or irritant contact dermatitis can also occur. There are also mentions of burns secondary to use of microwaves or defibrillators under the area where the disc was placed. Patch formulations release a high amount of drug, potentiating adverse effects. However, measurement of dose is hampered, especially in ointments, which become less safe when compared to patch. Abrupt suspension of nitroglycerin use can result in acute myocardial infarction, peripheral ischemia, and Raynaud's phenomenon.

Since topical nitrates are devoid of renal, gastrointestinal, and hematological adverse effects, they can be considered for the treatment of local pain, especially in patients contraindicated for NSAID use. ${ }^{63}$

\section{Other drugs}

Other pharmacological agents have been applied topically for several types of pain, such as clonidine and gabapentin. ${ }^{65,66}$ Some of these are sold over-the-counter, without medical prescription. The most recognized agents are described below.

\section{Glutamate receptor antagonist}

Ketamine is a parenteral anesthetic that provides analgesic effects in anesthetic subdoses. It is a noncompetitive $\mathrm{N}$-methyl-aspartate (NMDA) receptor antagonist with opioid activity. Topical ketamine enhances local anesthetic effects of bupivacaine in nociceptive and neuropathic pain. Its analgesic effects rely on glutamate receptor activity, voltagesensitive calcium channel blockage, interference with opioid receptors, and cholinergic and monoaminergic functions.

Indeed, ionotropic glutamate receptors are expressed on peripheral afferent nerve terminals and membranes of unmyelinated peripheral axons, in proportion to the rate of local inflammatory response. In animal models, the increase of NMDA and non-NMDA glutamate receptors is associated with hyperalgesia and allodynia. ${ }^{67,68}$

Ketamine hydrochloride is FDA-approved for intravenous and intramuscular use and comes in three forms: powder, liquid, and tablet. The main trademarks are Ketalar ${ }^{\mathbb{B}}$ (Pfizer Inc., New York, NY), Ketanest ${ }^{\circledR}$ (Pfizer Inc), and

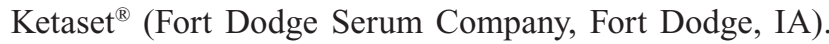
The analgesic response to intranasal, rectal, and transdermal routes of application have been tested in the treatment of refractory nociceptive and neuropathic pain. Transdermal preparations include hydrogel $1 \%$, organogel (known as Pluronic ${ }^{\circledR}$ lecithin organogel [PLO]. It is a liposomal phospholipid microemulsion), and oil-in-water $0.5 \%$ or $1 \%$ cream or emulsion.

Different vehicles and additives are being tested for the development of a transdermal therapeutic system of ketamine delivery, aiming to attain the concentration of the drug in serum. The hydrogel system provided the highest systemic level of circulating ketamine.

Small trials suggest that $0.5 \%-1 \%$ ketamine cream may benefit postoperative, sympathetically maintained, and cancer pain when applied topically. A case report described the efficacy of $0.5 \%$ ketamine hydrochloride cream associated with $2.5 \%$ amitriptyline hydrochloride that gave pain relief and improvement of quality of life in patients suffering from idiopathic proctodynia. ${ }^{69}$ Because ketamine is a phencyclidine analog and leads to psychomimetic effects, it is not used routinely as an anesthetic, although there has been a dangerous increase in recreational use of ketamine.

\section{Alpha-adrenoceptor antagonists}

Clonidine hydrochloride is available in $0.1,0.2$, and $0.3 \mathrm{mg}$ tablets, generally for oral treatment of systemic hypertension (Catapres ${ }^{\circledR}$; Boehringer-Inghelm Phamaceuticals Inc., Ridgefield, CT); however, extensive analgesic use of oral 
clonidine is limited by centrally mediated side effects (sedation, hypotension, and rebound hypertension).

When applied topically as creams or patches (Catapres $\left.\mathrm{TTS}^{\circledR}\right)$, it is a potent antinociceptive agent that produces central and peripheral analgesic effects by blocking the emerging pain signals at peripheral terminals via alpha-2 adrenoceptors without producing the undesirable central side effects observed in systemic administration. Nevertheless, there are reports of symptomatic worsening of hyperalgesia in the presence of peripheral nervous damage.

It has been used to control hyperalgesia present in complex regional pain syndromes, likely due to the reduction of presynaptic norepinephrine release from sympathetic nerves. ${ }^{65}$ This might explain the worsening of mechanical hyperalgesia that sympathetically maintained pain patients present when submitted to localized injection of norepinephrine. ${ }^{70}$

Topical administration of clonidine in mice was found to elicit analgesia, and the response suggests that the actions of clonidine are locally mediated. The ineffectiveness of topical ketamine to block topical clonidine antinociceptive tolerance suggests that peripheral NMDA receptors do not mediate local clonidine antinociceptive tolerance.

The transdermal therapeutic system for clonidine delivery consists of a multilayered film containing the active agent, designed for constant drug release over 7 days.

The system is $3.5 \mathrm{~cm}^{2}$ thick, $7.0 \times 10.5 \mathrm{~cm}^{2}$, composed of four consecutive layers: 1) backing layer of polyester and aluminum, 2) drug reservoir of clonidine, mineral oil, polyisobutylene, and colloidal silicon dioxide, 3) microporous polypropylene membrane that controls the rate of delivery, and 4) adhesive formulation of clonidine, mineral oil, polyisobutylene, and colloidal silicon dioxide.

\section{Cannabinoids}

Cannabinoids (delta8-tetrahydrocannabinol [delta8THC], cannabidiol [CBD], and cannabinol [CBN]), extracted from Cannabis sativa L, act at peripheral sites and yield analgesia through the action on $\mathrm{CB} 1$ and $\mathrm{CB} 2$ receptors. Dorsal root ganglia cells contain mRNA for $\mathrm{CB} 1$ receptors. Additionally, CB1 receptor agonists inhibit nerve growth factor and calcitonin gene-related peptide release. ${ }^{71}$ Local analgesic actions of agonists for CB2 receptors include the inhibition of mast cell function and inflammatory pain.

The topical administration of selective cannabinoid receptor agents has been demonstrated to have analgesic effects in animal models of inflammatory and neuropathic pain (formalin, carrageenan hyperalgesia, and partial nerve injury), especially for the control of breakthrough pain.
Transdermal cannabinoids were developed to blunt the side effects related to dose peaks and have a controlled release of active drug over a period of days. Intranasal cannabinoid sprays are used as a low-dose adjuvant to patches, and to warrant rapid absorption for systemic effects. Indeed, coadministration of agonists for both $\mathrm{CB} 1$ and $\mathrm{CB} 2$ receptors tends to potentiate analgesia. A 50/50 mixture of CBN and $\mathrm{CBD}$ in the form of transmucosal spray benefited central pain in multiple sclerosis. ${ }^{72}$ These findings support the development of other topical formulations or combinations of cannabinoids that could be devoid of central adverse effects.

These substances require further clinical trials for safety analysis before gaining a foothold in clinical practice. Central actions are still the main concern for this class of agents. In addition, transporting these drugs into human skin, opportunities for combined therapies, and appropriate vehicles need to be studied to improve transdermal delivery systems.

\section{Medication adherence}

Adherence is defined as the extent to which the patient's medication consumption matches the prescribed regimen. The impact of nonadherence varies across chronic illnesses, from minimal to significant. In many diseases, poor compliance to medication intake and medical advice threatens treatment outcomes.

Several classes of correlates of adherence to long-term medication regimens have recently been identified and can be separated into groups: patient (depression and substance abuse history), contextual (social support and socioeconomic status), clinician communication skills (patient-clinician relationship), disease (chronicity and symptom burden), health care delivery (wait for appointment and medications), and treatment regimen factors. ${ }^{73}$ Analysis of treatment adherence among chronic diseases fails and results are conflicting, due to substantial methodological variability of measurement. Although this is an impediment, it is possible to detect the primary factors of therapeutic regimen that directly influence patient adherence. ${ }^{73}$

Several studies suggest the advantage of simplification of the regimen complexity, usually by decreasing the pill burden and dosage, while a few conclude that there is no systematic difference between the effects of changing doses and other behavioral interventions. They researches argue that while the half-life of some drugs favors their management and relieves the impact of consuming several intakes per day, they do not always ensure patients' compliance. The World Health 
Table I Brief description of different strategies to increase treatment adherence in selected diseases

$\checkmark$ Patient awareness of breast cancer has increased, and physicians are required to provide information regarding treatment goals and adverse effects of drugs, which are frequent and disabling. These issues influence compliance. ${ }^{78}$

$\checkmark$ Among patients with cardiac failure, the main predictor for cardiac rehabilitation was the physician's endorsement of the effectiveness of program. Adherence increased when patients were actively referred, educated, highly self-motivated, and when programs were easily accessible. ${ }^{79}$

$\checkmark$ The major barriers for a good glycemic control include low efficacy of oral hypoglycemic drugs, fear of hypoglycemia, issues related to convenience of treatment (subcutaneous route, invasive blood sugar monitoring), poor access to health services, and lifestyle, leading to low adherence. ${ }^{80}$

$\checkmark$ Educational sessions, psychotherapeutic interventions, and phone prompts in community psychiatric services increase adherence of psychotic patients. $^{81}$

$\checkmark$ Factors associated with poor treatment adherence among patients undergoing renal replacement therapy or under dialysis are frequent dosing, patient's perception of treatment benefits, poor patient-physician communication, lack of motivation, low socioeconomic background. Strategies for compliance are not well established, but some are suggested: treatment regimen simplification, establishing a partnership with the patient, and education. ${ }^{82}$

$\checkmark$ The most striking barrier to medication adherence in multiple sclerosis is forgetfulness to take pills, coping with adverse effects, and perceived lack of efficacy. Validated strategies include good provider-patient relationship, continuous education, and reinforcement regarding the benefit of treatment. ${ }^{83}$

$\checkmark$ Nonadherence of chronic obstructive pulmonary disease in $40 \%-60 \%$ as opposed to asthmatic patients, who adhere to inhalers and rescue medication. Economic factors and health beliefs influence device selection. ${ }^{84}$

$\checkmark$ Long-term adherence for asymptomatic conditions such as arterial hypertension is $50 \%$. For these patients, adverse effects related to antihypertensive therapies and costs threaten treatment adherence. ${ }^{85}$ The most commonly used method to increase in compliance in dyslipidemia ${ }^{86}$ and hypertension ${ }^{87}$ is dosing schedule modification from twice to once a day dosing.

Organization claims the main barriers to adherence related to regimen factors are high dosing and side effects.

For short-term treatment or seasonal diseases, ${ }^{74,75}$ straightforward strategies and delivery of information to patients increased compliance, but the results are not consistent across all studies.

For long-term treatment of chronic diseases, a combination of strategies such as providing detailed instructions, close follow-up, reminders, rewards for successful compliance, family therapy, psychological therapy, crisis intervention, reinforcement, supportive care, and telephone follow-up seek to improve adherence and clinical outcomes, but they lack efficacy, despite major effort and applied resources. ${ }^{76}$ However, a review of the impact of dosing schedules in chronic illness pointed out that adherence was higher among patients taking once-daily medications compared to thrice or more frequent dosing, suggesting that simpler dosing regimens increase adherence. ${ }^{77}$ Indeed, the increasing availability of new formulations of medications, including extended-release and modified-release products, and formulations requiring weekly dosing may ease the regimens.

The challenges of treatment adherence have been investigated in several diseases, and each condition needs a different strategy, as shown in Table 1.

In acute pain, the symptomatic, episodic feature of the disease minimizes issues related to compliance. They are not the focus of recent research studies. Studies focused on chronic pain treatment are concerned with the appropriate dose to achieve analgesia while avoiding negative outcomes such as addiction, rather than checking adherence and dosing relationships or compliance. Old studies point out that, different from other nonpainful diseases, chronic pain patients preferred multiple doses daily for analgesics, suggesting the worry that their pain would be undertreated if fewer doses were taken. ${ }^{88}$ Because the majority of studies on chronic diseases indicate dosing simplification for better patient adherence, there is a need for new studies to be conducted in order to verify the findings above.

When chronic pain patients are asked about those which are the least acceptable adverse experiences related to a drug, they report central nervous system impairments. ${ }^{89}$ Topical agents are devoid of most systemic effects, but can only be used in specific conditions, and their effectiveness must be further elaborated. Therefore, for chronic or acute localized peripheral painful syndromes, topical preparations may contribute to patient adherence, due to low incidence of collateral effects and minimal number of daily applications.

\section{Conclusion}

Although only a small number of topical agents are available for use in peripheral and local conditions, increasing evidence supports the efficacy of such preparations for the relief of nociceptive or neuropathic pain. Overall, topical preparations have a good safety profile, which might benefit the elderly and pediatric populations and those presenting with intolerance to traditional treatments. Nevertheless, the quality of most of the studies examined is compromised by methodological heterogeneity, such as small sample size, and lack of control groups. It is also worth noting that this is not a systematic review of each 
class of topical drug, which prevents a definitive interpretation of the evidence regarding topical drug efficacy and the compliance of patients submitted to these preparations.

Despite the paucity of data, the intervention most likely to result in optimal adherence for the majority of patients is simplification of drug regimens. Pharmaceutical companies should be encouraged to develop medications that simplify treatment regimes, augment compliance, and reduce adverse effects. Patients would have a greater spectrum of treatment options if extended release compounds were developed to provide alternative routes of administration, such as topical delivery. The potential advantage of topical analgesic drugs is the minimization of both the presence of central side effects and the loss of efficacy of complex treatment regimens. The opportunity to develop topical drugs would also benefit pediatric, frail, and elderly populations.

However, due to the absence of specific compliance and adherence studies comparing topical treatment versus traditional routes in pain management, the role of topical preparations in patient adherence remains obscure.

\section{Acknowledgments}

The authors thank Dove Medical Press Ltd staff, Luciano Tasso (figures), and Patricia Lynn Fortner (proofreading).

\section{Disclosure}

The authors declare no conflict of interest.

\section{References}

1. Kreitler S, Niv D. Cognitive impairment in chronic pain. Pain Clinical Updates. 2007;XV(4):1-4.

2. Hróbjartsson A, Gøtzsche PC. Placebo interventions for all clinical conditions. Cochrane Database Syst Rev. 2010;1:CD003974.

3. Argoff CE. Targeted peripheral analgesics therapy for neuropathic pain. Curr Pain Headache Rep. 2004;8(3):199-204.

4. de Paula E, Cereda CM, Tofoli GR, Franz-Montan M, Fraceto LF, de Araújo DR. Drug delivery systems for local anesthetics. Recent Pat Drug Deliv Formul. 2010;4(1):23-34.

5. Byl NN. The use of ultrasound as an enhancer for transcutaneous drug delivery: phonophoresis. Phys Ther. 1995;75(6):539-553.

6. Tiwari SB, Pai RM, Udupa N. Influence of ultrasound on the percutaneous absorption of ketorolac tromethamine in vitro across rat skin. Drug Deliv. 2004;11(1):47-51.

7. Kozanoglu E, Basaran S, Guzel R, Guler-Uysal F. Short term efficacy of ibuprofen phonophoresis versus continuous ultrasound therapy in knee osteoarthritis. Swiss Med Wkly. 2003;133(23-24):333-338.

8. Sammeta SM, Murthy SN. "ChilDrive": a technique of combining regional cutaneous hypothermia with iontophoresis for the delivery of drugs to synovial fluid. Pharm Res. 2009;26(11): 2535-2540.

9. Motta AF, Borges Junior NG, da Fonseca JC, Tonussi CR. The antinociceptive effect of iontophoretic direct application of diclofenac to arthritic knee-joints of rats. Life Sci. 2003;73(15):1995-2004.
10. Başkurt F, Ozcan A, Algun C. Comparison of effects of phonophoresis and iontophoresis of naproxen in the treatment of lateral epicondylitis. Clin Rehabil. 2003;17(1):96-100.

11. Hassan AH, Ableitner A, Stein C, Herz A. Inflammation of the rat paw enhances axonal transport of opioid receptors in the sciatic nerve and increases their density in the inflamed tissue. Neuroscience. 1993; 55(1):185-195.

12. Cerchietti LC, Navigante AH, Körte MW, et al. Potential utility of the peripheral analgesic properties of morphine in stomatitis-related pain: a pilot study. Pain. 2003;105(1-2):265-273.

13. Varrassi G, Marinangeli F, Ciccozzi A, Iovinelli G, Facchetti G, Ciccone A. Intra-articular buprenorphine after knee arthroscopy. A randomised, prospective, double-blind study. Acta Anaesthesiol Scand. 1999;43(1):51-55.

14. Wrench IJ, Taylor P, Hobbs GJ. Lack of efficacy of intra-articular opioids for analgesia after day-case arthroscopy. Anaesthesia. 1996;51(10): 920-922.

15. Faymonville ME, Libbrecht D. Transdermal buprenorphine: a current overview of pharmacological and clinical data. Rev Med Liege. 2008; 63(11):671-676.

16. Hans G, Robert D. Transdermal buprenorphine - a critical appraisal of its role in pain management. J Pain Res. 2009;2:117-134.

17. Kress HG. Clinical update on the pharmacology, efficacy and safety of transdermal buprenorphine. Eur J Pain. 2009;13(3):219-230.

18. Tassinari D, Sartori S, Tamburini E, et al. Adverse effects of transdermal opiates treating moderate-severe cancer pain in comparison to longacting morphine: a meta-analysis and systematic review of the literature. $J$ Palliat Med. 2008;11(3):492-501.

19. Gibbs M. The role of transdermal fentanyl patches in the effective management of cancer pain. Int J Palliat Nurs. 2009;15(7):354-359.

20. Nüesch E, Rutjes AW, Husni E, Qelch V, Jüni P. Oral or transdermal opioids for osteoarthritis of the knee or hip. Cochrane Database Syst Rev. 2009;4:CD003115.

21. Hair PI, Keating GM, McKeage K. Transdermal matrix fentanyl membrane patch (matrifen): in severe cancer-related chronic pain. Drugs. 2008;68(14):2001-2009.

22. Herndon CM. Iontophoretic drug delivery system: focus on fentanyl. Pharmacotherapy. 2007;27(5):745-754.

23. Mayes S, Ferrone M. Fentanyl $\mathrm{HCl}$ patient-controlled iontophoretic transdermal system for the management of acute postoperative pain. Ann Pharmacother. 2006;40(12):2178-2186.

24. Zernikow B, Michel E, Anderson B. Transdermal fentanyl in childhood and adolescence: a comprehensive literature review. J Pain. 2007; 8(3):187-207.

25. Mitchell A, Smith HS. Applying partially occluded fentanyl transdermal patches to manage pain in pediatric patients. J Opioid Manag. 2010;6(4):290-294.

26. Houck CS, Sethna NF. Transdermal analgesia with local anesthetics in children: review, update and future directions. Expert Rev Neurother. 2005;5(5):625-634.

27. Lander JA, Weltman BJ, So SS. EMLA and amethocaine for reduction of children's pain associated with needle insertion. Cochrane Database Syst Rev. 2006;3:CD004236.

28. Briggs M, Nelson EA. Topical agents or dressings for pain in venous leg ulcers. Cochrane Database Syst Rev. 2010;4:CD001177.

29. Sawyer J, Febbraro S, Masud S, Ashburn MA, Campbell JC. Heated lidocaine/tetracaine patch (Synera, Rapydan) compared with lidocaine/ prilocaine cream (EMLA) for topical anaesthesia before vascular access. Br J Anaesth. 2009;102(2):210-215.

30. Soltesz S, Dittrich K, Teschendorf P, Fuss I, Molter G. Topical anesthesia before vascular access in children. Comparison of a warmthproducing lidocaine-tetracaine patch with a lidocaine-prilocaine patch. Anaesthesist. 2010;59(6):519-523.

31. Wasner G, Kleinert A, Binder A, Schattschneider J, Baron R. Postherpetic neuralgia: topical lidocaine is effective in nociceptor-deprived skin. J Neurol. 2005;252(6):677-686. 
32. Rowbotham MC, Davies PS, Verkempick C, Galer BS. Lidocaine patch: double-blind controlled study of a new treatment of post-herpetic neuralgia. Pain. 1996;65(1):39-44.

33. Galer BS, Rowbotham MC, Perander J, Friedman E. Topical lidocaine patch relieves postherpetic neuralgia more effectively than a vehicle topical patch: results of an enriched enrollment study. Pain. 1999; 80(3):533-538.

34. Devers A, Galer BS. Topical lidocaine patch relieves a variety of neuropathic pain conditions: an open-label study. Clin J Pain. 2000;16(3): 205-208.

35. Khaliq W, Alam S, Puri N. Topical lidocaine for the treatment of postherpetic neuralgia. Cochrane Database Syst Rev. 2007;2: CD004846.

36. Hedayati H, Parsons J, Crowther CA. Topically applied anaesthetics for treating perineal pain after childbirth. Cochrane Database Syst Rev. 2005;2:CD004223.

37. Wiles MD, Dobson SA, Moppett IK. The effect of a new topical local anaesthetic delivery system on forearm skin blood flow reactivity. Anaesthesia. 2010;65(2):178-183.

38. Gimbel J, Linn R, Hale M, Nicholson B. Lidocaine patch treatment in patients with low back pain: results of an open-label, nonrandomized pilot study. Am J Ther. 2005;12(4):311-319.

39. Fleming JA, O'Connor BD. Use of lidocaine patches for neuropathic pain in a comprehensive cancer centre. Pain Res Manag. 2009;14(5): 381-388

40. Davies PS, Galer BS. Review of lidocaine patch $5 \%$ studies in the treatment of postherpetic neuralgia. Drugs. 2004;64(9):937-947.

41. Rao P, Knaus EE. Evolution of nonsteroidal anti-inflammatory drugs (NSAIDs): cyclooxygenase (COX) inhibition and beyond. J Pharm Pharm Sci. 2008;11(2):81s-110s.

42. Massey T, Derry S, Moore RA, McQuay HJ. Topical NSAIDs for acute pain in adults. Cochrane Database Syst Rev. 2010;6:CD007402.

43. Altman R, Barkin RL. Topical therapy for osteoarthritis: clinical and pharmacologic perspectives. Postgrad Med. 2009;121(2):139-147.

44. Conaghan PG, Dickson J, Grant RL; Guideline Development Group. Care and management of osteoarthritis in adults: summary of NICE guidance. BMJ. 2008;336(7642):502-503.

45. Yang JH, Kim TY, Lee JH, Yoon SW, Yang KH, Shin SC. Antihyperalgesic and anti-inflammatory effects of ketorolac tromethamine gel using pulsed ultrasound in inflamed rats. Arch Pharm Res. 2008 31(4):511-517.

46. Petersen B, Rovati S. Diclofenac epolamine (Flector) patch: evidence for topical activity. Clin Drug Investig. 2009;29(1):1-9.

47. Barthel HR, Haselwood D, Longley S 3rd, Gold MS, Altman RD. Randomized controlled trial of diclofenac sodium gel in knee osteoarthritis. Semin Arthritis Rheum. 2009;39(3):203-212.

48. Altman RD, Dreiser RL, Fisher CL, Chase WF, Dreher DS, Zacher J. Diclofenac sodium gel in patients with primary hand osteoarthritis: a randomized, double-blind, placebo-controlled trial. J Rheumatol. 2009;36(9):1991-1999.

49. Towheed TE. Pennsaid therapy for osteoarthritis of the knee: a systematic review and metaanalysis of randomized controlled trials. J Rheumatol. 2006;33(3):567-573.

50. Rainsford KD, Kean WF, Ehrlich GE. Review of the pharmaceutical properties and clinical effects of the topical NSAID formulation, diclofenac epolamine. Curr Med Res Opin. 2008;24(10): 2967-2992.

51. Mazières B. Topical ketoprofen patch. Drugs RD. 2005;6(6):337-344.

52. Rother M, Lavins BJ, Kneer W, Lehnhardt K, Seidel EJ, Mazgareanu S. Efficacy and safety of epicutaneous ketoprofen in Transfersome (IDEA-033) versus oral celecoxib and placebo in osteoarthritis of the knee: multicentre randomised controlled trial. Ann Rheum Dis. 2007;66(9):1178-1183.

53. Stucki G, Rother M; European IDEA-033 Study Group. Randomized, double-blind, placebo-controlled study of three dosages of epicutaneously applied IDEA-033 (keteprofen in Transfersome) in patients with osteoarthritis of the knee. Ann Rheum Dis. 2007;66 Suppl 2:S510.
54. Kneer W, Rother I, Rother M, Seidel E; IDEA-033-III-01 Study Group. A multiple-dose, open-label, safety, compliance, and usage evaluation study of epicutaneously applied Diractin (ketoprofen in Transfersome) in joint/musculoskeletal pain or soft tissue inflammation. Curr Drug Saf. 2009;4(1):5-10.

55. Whitefield M, O'Kane CJ, Anderson S. Comparative efficacy of a proprietary topical ibuprofen gel and oral ibuprofen in acute soft tissue injuries: a randomized, double-blind study. J Clin Pharm Ther. 2002; 27(6):409-417.

56. Trnavský K, Fischer M, Vögtle-Junkert U, Schreyger F. Efficacy and safety of 5\% ibuprofen cream treatment in knee osteoarthritis. Results of a randomized, double-blind, placebo-controlled study. J Rheumatol. 2004;31(3):565-572.

57. Brunton LL, Lazo JS, Parker KL, editors. Goodman and Gilman's The Pharmacological Basis of Therapeutics. 11th ed. New York, NY: McGraw-Hill; 2006.

58. Mason L, Moore RA, Derry S, Edwards JE, McQuay HJ. Systematic review of topical capsaicin for the treatment of chronic pain. $B M J$. 2004;328(7446):991-995

59. Derry S, Lloyd R, Moore RA, McQuay HJ. Topical capsaicin for chronic neuropathic pain in adults. Cochrane Database Syst Rev. 2009;4: CD007393.

60. Ellison N, Loprinzi CL, Kugler J, et al. Phase III placebo-controlled trial of capsaicin cream in the management of surgical neuropathic pain in cancer patients. J Clin Oncol. 1997;15(8):2974-2980.

61. Morgenlander JC, Hurwitz BJ, Massey EW. Capsaicin for the treatment of pain in Guillain-Barré syndrome. Ann Neurol. 1990;28(2):199.

62. Hempenstall K, Nurmikko TJ, Johnson RW, A'Hern RP, Rice AS. Analgesic therapy in postherpetic neuralgia: a quantitative systematic review. PLoS Med. 2005;2(7):e164.

63. Aronson JK, editor. Meyler's Side Effects of Drugs - The International Encyclopedia of Adverse Drug Reactions and Interactions. 15th ed. Amsterdam, The Netherlands: Elsevier Science; 2005.

64. Cumpston M, Johnston RV, Wengier L, Buchbinder R. Topical glyceryl trinitrate for rotator cuff disease. Cochrane Database Syst Rev. 2009;3: CD006355.

65. Davis KD, Treede RD, Raja SN, Meyer RA, Campbell JN. Topical application of clonidine relieves hyperalgesia in patients with sympathetically maintained pain. Pain. 1991;47(3):309-317.

66. Epstein JB, Grushka M, Le N. Topical clonidine for orofacial pain: a pilot study. J Orofac Pain. 1997;11(4):346-352.

67. Ushida T, Tani T, Kanbara T, Zinchuk VS, Kawasaki M, Yamamoto H. Analgesic effects of ketamine ointment in patients with complex regional pain syndrome type 1. RegAnesth Pain Med. 2002;27(5): 524-528.

68. Kronenberg RH. Ketamine as an analgesic: parenteral, oral, rectal, subcutaneous, transdermal and intranasal administration. J Pain Palliat Care Pharmacother. 2002;16(3):27-35.

69. Lehman JS, Sciallis GF. Effective use of topical amitriptyline hydrochloride $2.5 \%$ and ketamine hydrochloride $0.5 \%$ for analgesia in refractory proctodynia. J Drugs Dermatol. 2008;7(9):887-889.

70. Ali Z, Raja SN, Wesselmann U, Fuchs PN, Meyer RA, Campbell JN. Intradermal injection of norepinephrine evokes pain in patients with sympathetically maintained pain. Pain. 2000;88(2):161-168.

71. Richardson JD, Kilo S, Hargreaves KM. Cannabinoids reduce hyperalgesia and inflammation via interaction with peripheral CB1 receptors. Pain. 1998;75(1):111-119.

72. Rog DJ, Nurmikko TJ, Friede T, Young CA. Randomized, controlled trial of cannabis-based medicine in central pain in multiple sclerosis Neurology. 2005;65(6):812-819.

73. Ingersoll KS, Cohen J. The impact of medication regimen factors on adherence to chronic treatment: a review of literature. J Behav Med. 2008;31(3):213-224.

74. Henry A, Batey RG. Enhancing compliance not a prerequisite for effective eradication of Helicobacter pylori: the HelP study. Am J Gastroenterol. 1999;94(3):811-815. 
75. Gani F, Pozzi E, Crivellaro MA, et al. The role of patient training in the management of seasonal rhinitis and asthma: clinical implications. Allergy. 2001;56(1):65-68.

76. Haynes RB, Ackloo E, Sahota N, McDonald HP, Yao X. Interventions for enhancing medication adherence. Cochrane Database Syst Rev. 2008;2:CD000011.

77. Claxton AJ, Cramer J, Pierce C. A systematic review of the associations between dose regimens and medication compliance. Clin Ther. 2001; 23(8):1296-1310.

78. Harbeck N, Haidinger R. The patient experience. Breast Cancer Res Treat. 2007;105 Suppl 1:91-103.

79. Jackson L, Leclerc J, Erskine Y, Linden W. Getting the most out of cardiac rehabilitation: a review of referral and adherence predictors. Heart. 2005;91(1):10-14.

80. Barnett AH. Treating to goal: challenges of current management. Eur J Endocrinol. 2004;151 Suppl 2:T3-T7.

81. Nosé M, Barbui C, Gray R, Tansella M. Clinical interventions for treatment non-adherence in psychosis: meta-analysis. Br J Psychiatry. 2003;183:197-206.

82. Loghman-Adham M. Medication noncompliance in patients with chronic disease: issues in dialysis and renal transplantation. Am J Manag Care. 2003;9(2):155-171.
83. Costello K, Kennedy P, Scanzillo J. Recognizing nonadherence in patients with multiple sclerosis and maintaining treatment adherence in the long term. Medscape J Med. 2008;10(9):225.

84. Restrepo RD, Alvarez MT, Wittnebel LD, et al. Medication adherence issues in patients treated for COPD. Int J Chron Obstruct Pulmon Dis. 2008;3(3):371-384.

85. After the diagnosis: adherence and persistence with hypertension therapy. Am J Manag Care. 2005;11(13 Suppl):S395-S399.

86. Brown BG, Bardsley J, Poulin D, et al. Moderate dose, three-drug therapy with niacin, lovastatin, and colestipol to reduce lowdensity lipoprotein cholesterol $<100 \mathrm{mg} / \mathrm{dl}$ in patients with hyperlipidemia and coronary artery disease. Am J Cardiol. 1997;80(2): 111-115.

87. Girvin B, McDermott BJ, Johnston GD. A comparison of enalapril $20 \mathrm{mg}$ once daily vs $10 \mathrm{mg}$ twice daily in terms of blood pressure lowering and patient compliance. J Hypertens. 1999;17(11):1627-1631.

88. Kubacka RT, Juhl RP. Attitudes of patients with hypertension or arthritis toward the frequency of medication administration. Am J Hosp Pharm. 1985;42(11):2499-2501.

89. Edwards JE. Determination of analgesic efficacy and harm in acute postoperative pain using systematic review methods. Thesis. Oxford, UK: University of Oxford; 2000.
Journal of Pain Research

\section{Publish your work in this journal}

The Journal of Pain Research is an international, peer-reviewed, open access, online journal that welcomes laboratory and clinical findings in the fields of pain research and the prevention and management of pain. Original research, reviews, symposium reports, hypothesis formation and commentaries are all considered for publication.

\section{Dovepress}

The manuscript management system is completely online and includes a very quick and fair peer-review system, which is all easy to use. Visit http://www.dovepress.com/testimonials.php to read real quotes from published authors. 